\title{
Torsional magnetic tube waves in stellar convection zones
}

\section{Analysis of wave generation and application to the Sun}

\author{
M. W. Noble ${ }^{1}$, Z. E. Musielak ${ }^{1,2}$, and P. Ulmschneider ${ }^{2}$ \\ 1 Department of Physics, University of Texas at Arlington, Arlington, TX 76019, USA \\ 2 Institut für Theoretische Astrophysik der Universität Heidelberg, Tiergartenstr. 15, 69121 Heidelberg, Germany \\ Received 15 April 2003 / Accepted 1 July 2003

\begin{abstract}
An analytic approach to the generation of torsional magnetic tube waves in stellar convection zones is presented. The waves are produced in a thin, vertically oriented magnetic flux tube embedded in a magnetic field-free, turbulent and compressible external medium and are excited by external turbulent flows. A theory for this interaction is developed and used to compute the wave energy spectra and fluxes carried by torsional tube waves in the solar atmosphere. We find that these tube waves have a characteristic cutoff frequency.
\end{abstract}

Key words. methods: numerical - Sun: chromosphere - Sun: corona - Sun: magnetic fields - MHD - waves

\section{Introduction}

In the magnetic regions of the solar and stellar atmospheres, isolated small-scale magnetic structures (flux tubes) are present (e.g., Solanki 1993; Saar 1996). These tubes are rooted in the solar and stellar convection zones where they interact with the external turbulent motions. This interaction leads to the generation of waves that carry energy along the tubes to the overlying atmosphere (Spruit \& Roberts 1983). It has been shown that three different types of waves can be supported by these flux tubes, namely, longitudinal, transverse and torsional tube waves (see Spruit 1981, 1982). These waves can dissipate the carried energy and heat the atmosphere to temperatures higher than those corresponding to the radiative equilibrium. As a result of this heating, the Sun and other late-type stars are sources of chromospheric activity (e.g., Linsky 1991).

Significant theoretical and observational efforts have been made to estimate the amount of energy carried by longitudinal (Musielak et al. 1989, 1995; Ulmschneider \& Musielak 1998; Ulmschneider et al. 2001) and transverse (Muller et al. 1989, 1994; Choudhuri et al. 1993a,b; Huang et al. 1995; Musielak \& Ulmschneider 2001) tube waves. Analytical studies are based on the Lighthill-Stein theory of sound generation (Lighthill 1952; Stein 1967), which has been modified to include the magnetic flux tube structure, and also its treatment of turbulence has been improved (Musielak et al. 1994). Among the three different tube waves, only the generation of longitudinal and transverse tube waves have been analytically investigated (Musielak et al. 1995; Musielak \& Ulmschneider 2001), and the resulting stellar wave energy spectra and fluxes have been

Send offprint requests to: $\mathrm{P}$. Ulmschneider, e-mail: ulmschneider@ita.uni-heidelberg.de calculated (Musielak et al. 2000, 2002; Musielak \& Ulmschneider 2002a,b). To complement this series of analytical studies, we now investigate the efficiency of the generation of torsional tube waves in the solar and stellar convection zones.

Recently, Ulmschneider et al. (2001) and Fawzy et al. (2002a,b) have shown that longitudinal and transverse tube waves do not carry enough energy to heat the upper chromospheric layers of active and moderately active stars, and suggested that the energy carried by torsional tube waves must be taken into account. These authors have constructed theoretical models of stellar chromospheres based on the heating of non-magnetic regions by acoustic waves and magnetic regions by longitudinal and transverse tube waves (see also Buchholz et al. 1998; Cuntz et al. 1998, 1999). The models have been used to predict theoretically the level of chromospheric activity. By comparing the theoretical predictions with observations, the authors concluded that other heating mechanisms must be included in their models. Among many different heating mechanisms (e.g., Narain \& Ulmschneider 1996), the most promising additional heating mechanisms are those that heat by reconnection events and by torsional tube waves. In order to include dissipation of torsional tube waves into these theoretical chromospheric models, the initial wave energy fluxes carried by these waves into the solar and stellar atmospheres must be known. The main goal of this series of papers is therefore to calculate the spectra and wave energy fluxes of torsional Alfvén waves in magnetic flux tubes.

We consider a thin magnetic flux tube that is embedded in a non-magnetic, compressible and convectively unstable (turbulent) medium. We assume that the tube is oriented vertically and that there are no turbulent motions inside the tube. 
This means that the only source of torsional tube waves is the turbulent flow outside the tube; the flow is assumed to be subsonic, so that the generated waves have small amplitudes. The fact that the tube is vertical and the generated waves are linear allows us to separate the excitation of torsional tube waves from the other two tube wave modes. It must be also mentioned that our approach automatically includes the correlation effects of cancellation and amplification that are always present when the magnetic flux tube is excited at many points along its length. As a result of the interaction between the flux tube and the external turbulent motions torsional tube waves are excited and the wave energy carried by these waves propagates outward where it is dissipated in the overlying atmosphere. The processes of the wave propagation and dissipation will be discussed in separate papers.

In this paper, we develop a theory describing the generation of torsional tube waves in solar and stellar convection zones, and apply it to the Sun; the wave energy spectra and fluxes for other late-type stars of Population I and II will be presented in two forthcoming papers. Our approach is analytical and its first step is to derive and solve the inhomogeneous wave equation, and then obtain the basic expressions for the wave energy spectra and fluxes (see Sect. 2). Application of the obtained results to the Sun and their discussion are given in Sect. 3. The main conclusions of the paper are summarized in Sect. 4 .

\section{Inhomogeneous wave equation and energy fluxes}

\subsection{Model and assumptions}

We consider an isolated magnetic flux tube, which is assumed to be thin and with a circular cross-section. The tube is embedded in a magnetic field-free, turbulent, compressible and isothermal external medium. The tube is in temperature equilibrium with the external medium and it remains vertically oriented under the assumption that there are strong buoyancy forces in the upper layers of the convection zones. Because of this vertical orientation, the three fundamental (longitudinal, transverse and torsional) tube wave modes can be separated and, as a result, the generation of torsional tube waves can be treated independently from the other two tube waves. It is assumed that the torsional waves are excited only by the external turbulent motions and that there are no fluid motions inside the tube that could affect the generation and propagation of these waves. Our approach is limited to subsonic turbulence, which means that the turbulent Mach number $M_{\mathrm{t}}=u_{\mathrm{t}} / c_{\mathrm{S}}<1$, where $u_{\mathrm{t}}$ is the rms turbulent velocity and $c_{\mathrm{S}}$ is the sound speed both inside and outside the flux tube. This subsonic turbulence leads to the generation of linear torsional waves, which show negligible coupling to other tube wave modes in the region of wave excitation. However, this coupling may become much more effective in higher atmospheric layers where nonlinear effects are important (e.g., Ulmschneider et al. 1991; Hollweg et al. 1982).

To describe torsional tube waves, we adopt a cylindrical coordinate system $(R, \phi, z)$ and determine the height dependence of the internal tube parameters by using the thin flux tube approximation. This allows us to write the magnetic field strength, gas density and gas pressure inside the tube as $\boldsymbol{B}_{\mathrm{o}}=B_{\mathrm{o}}(z) \hat{z}, \rho_{\mathrm{o}}=\rho_{\mathrm{o}}(z)$ and $p_{\mathrm{o}}=p_{\mathrm{o}}(z)$, respectively, where the subscript "o" indicates internal parameters and $\hat{z}$ is the unit vector in the $z$-direction. Outside the tube, the magnetic field is zero, $B_{\mathrm{e}}=0$, the gas density and pressure are expressed as $\rho_{\mathrm{e}}=\rho_{\mathrm{e}}(z)$ and $p_{\mathrm{e}}=p_{\mathrm{e}}(z)$, respectively, with the subscript "e" denoting external parameters. A plane-parallel and isothermal atmosphere is assumed with uniform gravitational acceleration given by $\boldsymbol{g}=-g \hat{z}$.

By assuming that the tube axis is always oriented along the $z$-axis, the torsional waves are completely described by perturbations of the magnetic field $\boldsymbol{b}=b_{\phi}(z, t) \hat{\boldsymbol{\phi}}$ and tube wave velocity given by $\boldsymbol{v}=v_{\phi}(z, t) \hat{\boldsymbol{\phi}}$ with $\hat{\phi}$ being the unit vector in the $\phi$-direction; note that the tube is being twisted by the external turbulent motions, however, no fluid motions in the external medium are due to torsional oscillations of the tube. This makes the total magnetic field for the purely torsional mode $\boldsymbol{B}=B_{0} \hat{z}+b_{\phi} \hat{\phi}$. Because the considered torsional tube waves are linear, the turbulent density and pressure perturbations associated with these waves can be neglected $(\delta \rho=\delta p=0)$. This allows the internal total gas density $\rho$ and total gas pressure $p$ to be replaced with their equilibrium values $\rho_{\mathrm{o}}$ and $p_{\mathrm{o}}$, and the waves to be considered incompressible, which means that $\nabla \cdot v=0$.

\subsection{Wave equation}

If the magnetic flux tube is to remain stable there must be a balance of the internal and external pressures across the tube boundary. The sum of the internal gas and magnetic pressures must be equal to the external gas pressure at the interface: $p_{\mathrm{o}}+B_{\mathrm{o}}^{2} / 8 \pi=p_{\mathrm{e}}+\delta p_{\mathrm{t}}+\delta p_{\mathrm{w}}$, where $\delta p_{\mathrm{t}}$ and $\delta p_{\mathrm{w}}$ are pressure fluctuations in the external medium due to turbulence and external acoustic waves. Since the latter are compressible waves, we neglect their contribution to the generation of purely incompressible torsional tube waves by taking $\delta p_{\mathrm{w}}<\delta \delta p_{\mathrm{t}}$ which also implies that $\delta \rho_{\mathrm{w}}<\delta \rho_{\mathrm{t}}$ (Spruit 1982; Musielak $\&$ Ulmschneider 2001). Thus, the horizontal pressure balance can be written as

$p_{\mathrm{o}}+\frac{B_{\mathrm{o}}^{2}}{8 \pi}=p_{\mathrm{e}}+\delta p_{\mathrm{t}}$

The momentum equation for a magnetic flux tube oscillating with velocity $\boldsymbol{v}$ is given by

$\rho_{\mathrm{o}} \frac{\partial \boldsymbol{v}}{\partial t}+\rho_{\mathrm{o}}(\boldsymbol{v} \cdot \nabla) \boldsymbol{v}=-\nabla p_{\mathrm{o}}+\frac{1}{c} \boldsymbol{J} \times \boldsymbol{B}+\rho_{\mathrm{o}} \boldsymbol{g}$,

where the link between fluid equations and electromagnetic equations is $\boldsymbol{J} \times \boldsymbol{B}=\frac{c}{4 \pi}(\nabla \times \boldsymbol{B}) \times \boldsymbol{B}$, and where $c$ is the light speed. For linear waves, Eq. (2) can be expanded and written as

$$
\begin{aligned}
\rho_{\mathrm{o}} \frac{\partial \boldsymbol{v}}{\partial t}= & -\nabla\left(p_{\mathrm{o}}+\frac{B_{\mathrm{o}}^{2}}{8 \pi}\right)+\frac{1}{4 \pi}\left(\boldsymbol{B}_{\mathrm{o}} \cdot \nabla\right) \boldsymbol{B}_{\mathrm{o}} \\
& +\frac{1}{4 \pi}\left[\left(\nabla \times \boldsymbol{B}_{\mathrm{o}}\right) \times \boldsymbol{b}+(\nabla \times \boldsymbol{b}) \times \boldsymbol{B}_{\mathrm{o}}\right]+\rho_{0} \boldsymbol{g} .
\end{aligned}
$$

Using the conditions specified above, the linearized momentum equation for the external medium with velocity $\boldsymbol{u}$ is

$\rho_{\mathrm{te}} \frac{\partial \boldsymbol{u}}{\partial t}+\nabla p_{\mathrm{te}}-\rho_{\mathrm{te}} \boldsymbol{g}=0$ 
where $\rho_{\mathrm{te}}=\rho_{\mathrm{e}}+\delta \rho_{\mathrm{t}}$ and $p_{\mathrm{te}}=p_{\mathrm{e}}+\delta p_{\mathrm{t}}$ contain fluctuating components caused by the turbulence. Again, since the tube is twisted but does not move horizontally, no motion is due to the tube in the external medium and Eq. (4) can be written in the following form:

$\nabla\left(p_{\mathrm{e}}+\delta p_{\mathrm{t}}\right)=-\delta \rho_{\mathrm{t}} \frac{\partial \boldsymbol{u}}{\partial t}+\left(\rho_{\mathrm{e}}+\delta \rho_{\mathrm{t}}\right) \boldsymbol{g}$,

with $\rho_{\mathrm{e}}(\partial \boldsymbol{u} / \partial t)=0$ because the external medium is in dynamical steady-state.

Combining Eqs. (1), (3) and (5), yields

$$
\begin{aligned}
\rho_{\mathrm{o}} \frac{\partial \boldsymbol{v}}{\partial t}-\delta \rho_{\mathrm{t}} \frac{\partial \boldsymbol{u}}{\partial t}= & \frac{1}{4 \pi}\left(\boldsymbol{B}_{\mathrm{o}} \cdot \nabla\right) \boldsymbol{B}_{\mathrm{o}}+\left(\rho_{\mathrm{o}}-\rho_{\mathrm{e}}\right) \boldsymbol{g}-\delta \rho_{\mathrm{t}} \boldsymbol{g} \\
& +\frac{1}{4 \pi}\left[\left(\nabla \times \boldsymbol{B}_{\mathrm{o}}\right) \times \boldsymbol{b}+(\nabla \times \boldsymbol{b}) \times \boldsymbol{B}_{\mathrm{o}}\right] .
\end{aligned}
$$

Since $\boldsymbol{B}_{\mathrm{o}}$ is a function of $z$ only, taking the $\phi$-component of Eq. (6) gives

$$
\begin{aligned}
\rho_{\mathrm{o}}\left(\frac{\partial \boldsymbol{v}}{\partial t}\right)_{\phi}-\delta \rho_{\mathrm{t}}\left(\frac{\partial \boldsymbol{u}}{\partial t}\right)_{\phi} & = \\
& \frac{1}{4 \pi}\left[\left(\nabla \times \boldsymbol{B}_{\mathrm{o}}\right) \times \boldsymbol{b}+(\nabla \times \boldsymbol{b}) \times \boldsymbol{B}_{\mathrm{o}}\right]_{\phi} .
\end{aligned}
$$

Defining $(\boldsymbol{v})_{\phi}=v_{\phi}$ and $(\boldsymbol{u})_{\phi}=u_{\phi}$, and rearranging the terms, Eq. (7) can be written as

$\frac{\partial v_{\phi}}{\partial t}-\frac{B_{\mathrm{o}}}{4 \pi \rho_{\mathrm{o}}} \frac{\partial b_{\phi}}{\partial z}=\frac{\delta \rho_{\mathrm{t}}}{\rho_{\mathrm{o}}} \frac{\partial u_{\phi}}{\partial t}$.

To eliminate $b_{\phi}$ from the above equation, it is necessary to take the induction equation into consideration. Assuming that Ohm's law holds and displacements current are negligible when compared to conduction currents, we obtain

$\left(\frac{\partial \boldsymbol{b}}{\partial t}\right)_{\phi}-\left[\nabla \times\left(\boldsymbol{v} \times \boldsymbol{B}_{\mathrm{o}}\right)\right]_{\phi}=0$,

where only linear terms are kept. From Eq. (9), we find

$b_{\phi}=B_{\mathrm{o}}\left(\frac{\partial}{\partial t}\right)^{-1}\left(\frac{\partial v_{\phi}}{\partial z}\right)$.

Combining Eqs. (8), (10), and taking $\partial / \partial t$ of the result yields

$\frac{\partial^{2} v_{\phi}}{\partial t^{2}}-c_{\mathrm{A}}^{2} \frac{\partial^{2} v_{\phi}}{\partial z^{2}}-c_{\mathrm{A}}^{2} \frac{B_{\mathrm{o}}^{\prime}}{B_{\mathrm{o}}} \frac{\partial v_{\phi}}{\partial z}=\frac{\partial}{\partial t}\left[\frac{\delta \rho_{\mathrm{t}}}{\rho_{\mathrm{o}}}\left(\frac{\partial u_{\phi}}{\partial t}\right)\right]$,

where $B_{\mathrm{o}}^{\prime}=\mathrm{d} B_{\mathrm{o}} / \mathrm{d} z$ and

$c_{\mathrm{A}}^{2}=\frac{B_{\mathrm{o}}^{2}}{4 \pi \rho_{\mathrm{o}}}$,

which remains constant along the tube because of the thin flux tube approximation.

The physical properties of torsional tube waves and the fact that their characteristic velocity $c_{\mathrm{A}}$ is the same as the Alfvén velocity clearly imply that these waves are very similar to Alfvén waves in ideal MHD with uniform magnetic fields. There are also similarities between transverse tube waves and Alfvén waves, however, the propagation velocity of the former is affected by the presence of the external medium (see Spruit 1981) and, therefore, it differs from the Alfvén velocity. Longitudinal tube waves are essentially acoustic waves guided by the tube magnetic field lines, so they resemble slow MHD waves propagating along the field lines; note that no mode of the flux tube geometry corresponds to fast MHD waves.

From the definition of the Alfvén velocity (see Eq. (12)) and the fact that $c_{\mathrm{A}}=$ const. in the approach considered here, we obtain

$\frac{B_{\mathrm{o}}^{\prime}}{B_{\mathrm{o}}}=\frac{1}{2} \frac{\rho_{\mathrm{o}}^{\prime}}{\rho_{\mathrm{o}}}$,

where $\rho_{\mathrm{o}}^{\prime}=\mathrm{d} \rho_{\mathrm{o}} / \mathrm{d} z$. In addition, we have

$\frac{\rho_{\mathrm{O}}^{\prime}}{\rho_{\mathrm{o}}}=-\frac{1}{H}$,

with $H$ being the density (pressure) scale height of the internal and external atmosphere. Hence, Eq. (11) can be written as

$\left[\frac{\partial^{2}}{\partial t^{2}}-c_{\mathrm{A}}^{2} \frac{\partial^{2}}{\partial z^{2}}+\frac{c_{\mathrm{A}}^{2}}{2 H} \frac{\partial}{\partial z}\right] v_{\phi}(z, t)=S_{\phi}(z, t)$,

and the source function is

$S_{\phi}(z, t)=\frac{\partial}{\partial t}\left[\frac{\delta \rho_{\mathrm{t}}}{\rho_{\mathrm{o}}}\left(\frac{\partial u_{\phi}}{\partial t}\right)\right]$.

In the case of no turbulent external motions, the source function is zero and the wave equation describes freely propagating torsional tube waves.

Now, it must be noted that the derived inhomogeneous wave Eq. (15) has similar form to that obtained for transverse tube waves (see Musielak \& Ulmschneider 2001, and their Eq. (12)). The presence of the first order derivative of $v_{\phi}$ with respect to height implies that a cutoff frequency can be introduced for torsional tube waves propagating along a diverging magnetic flux tube (see Sect. 2.3). As shown by Eqs. (11), (13) and (14), the existence of this cutoff is caused by the gradient of the magnetic field $B_{0}^{\prime}$. Obviously, this cutoff frequency would not be present if a homogeneous slab would be considered instead of a flux tube with exponentially diverging magnetic fields.

\subsection{Klein-Gordon equation and the cutoff frequency}

To derive the cutoff frequency for torsional tube waves, we cast Eq. (15) into its Klein-Gordon form by using the following transformation:

$v_{\phi}=\rho_{\mathrm{o}}^{-1 / 4} v$,

which yields

$\left(\frac{\partial^{2}}{\partial t^{2}}-c_{\mathrm{A}}^{2} \frac{\partial^{2}}{\partial z^{2}}+\Omega_{\mathrm{T}}^{2}\right) v=S_{\mathrm{t}}(z, t)$,

where $S_{\mathrm{t}}(z, t)=\rho_{\mathrm{o}}^{1 / 4} S_{\phi}(z, t)$ and the cutoff frequency $\Omega_{\mathrm{T}}$ for torsional tube waves is defined as

$\Omega_{\mathrm{T}}=\frac{c_{\mathrm{A}}}{4 H}$.

In order to avoid conflicting notations compared to our previous work (e.g. Musielak et al. 1995), the cutoff frequency of 
longitudinal tube waves, sometimes called Defouw frequency, instead of termed $\Omega_{\mathrm{t}}$ is from now on denoted by $\Omega_{\mathrm{D}}$. Since both the propagation velocity $c_{\mathrm{A}}$ of torsional waves and the scale height $H$ are constant along the tube, the cutoff frequency $\Omega_{\mathrm{T}}$ is a global cutoff as it is the same along the entire length of the (exponentially spreading) tube. Torsional waves are propagating waves when their frequency $\omega$ satisfies the condition $\omega>\Omega_{\mathrm{T}}$, and they are evanescent when $\omega \leq \Omega_{\mathrm{T}}$. It is interesting to note that in the previous work (e.g., Spruit 1981, 1982; also Roberts \& Ulmschneider 1997, and references therein), the cutoff frequency for torsional waves has not been explicitly introduced. Our results clearly show that this cutoff must always be taken into account when magnetic flux tube structures embedded in the solar and stellar atmospheres are considered.

The definitions of the cutoff frequency for torsional, $\Omega_{\mathrm{T}}$, and transverse (kink), $\Omega_{\mathrm{K}}$ (Spruit 1981; also Musielak \& Ulmschneider 2001), tube waves are very similar in form, however, the ratio of these cutoffs is

$\frac{\Omega_{\mathrm{T}}}{\Omega_{\mathrm{K}}}=\frac{c_{\mathrm{A}}}{c_{\mathrm{K}}}=\sqrt{1+\frac{\rho_{\mathrm{o}}}{\rho_{\mathrm{e}}}}=\sqrt{(2 \beta+1) / \beta}$,

where $\beta=8 \pi p_{\mathrm{o}} / B_{\mathrm{o}}^{2}$. Here $c_{\mathrm{K}}=c_{\mathrm{A}} \sqrt{\rho_{\mathrm{o}} /\left(\rho_{\mathrm{o}}+\rho_{\mathrm{e}}\right)}$ is the kink speed. For $\beta>>1$, we have $\Omega_{\mathrm{T}} / \Omega_{\mathrm{K}} \approx \sqrt{2}$, and for $\beta<<1$, one finds $\Omega_{\mathrm{T}} / \Omega_{\mathrm{K}} \approx 1 / \sqrt{\beta}$. This clearly shows that torsional tube waves always propagate faster than transverse tube waves, and also that the cutoff frequency for the former is higher than for the latter (see Fig. 2); the higher cutoff implies that the wave energy spectra for torsional tube waves are not as broad as those for transverse tube waves (see Sect. 3).

We may also compare the torsional and longitudinal, $\Omega_{\mathrm{D}}$ (see Defow 1976; also Musielak et al. 1995), cutoff frequencies. The ratio of these two cutoffs is

$$
\frac{\Omega_{\mathrm{T}}}{\Omega_{\mathrm{D}}}=\sqrt{\frac{2+\gamma \beta}{\gamma \beta}\left(9-\frac{8}{\gamma}+8 \beta \frac{\gamma-1}{\gamma}\right)^{-1}},
$$

with $\Omega_{\mathrm{T}} / \Omega_{\mathrm{D}} \approx \sqrt{\gamma / 8 \beta(\gamma-1)} \approx 0.56 / \sqrt{\beta}$ when $\beta>>1$ and $\Omega_{\mathrm{T}} / \Omega_{\mathrm{D}} \approx \sqrt{2 /(9 \gamma-8) \beta} \approx 0.53 / \sqrt{\beta}$ when $\beta<<1$.

This shows that the ratio is very sensitive to the value of plasma $\beta$; for typical values of $\beta$ considered here $\Omega_{\mathrm{T}}$ is always lower than $\Omega_{\mathrm{D}}$ (see Fig. 2), which implies that the wave energy spectra for torsional tube waves are always broader than those obtained for longitudinal tube waves (Musielak et al. 1995). Finally, the comparison of the cutoff frequency for torsional and acoustic, $\Omega_{\mathrm{S}}$ (Lamb 1908), waves shows that $\Omega_{\mathrm{T}}$ is always marginally lower than $\Omega_{\mathrm{S}}$ and, therefore, the generated acoustic wave energy spectra are broader (e.g., Ulmschneider et al. 1996).

\subsection{Source function}

We now consider the source function given by

$S_{\mathrm{t}}(z, t)=\rho_{\mathrm{o}}^{1 / 4} \frac{\partial}{\partial t}\left[\frac{\delta \rho_{\mathrm{t}}}{\rho_{\mathrm{o}}}\left(\frac{\partial u_{\phi}}{\partial t}\right)\right]$.
The continuity equation for the external medium allows $\delta \rho_{\mathrm{t}}$ to be expressed as a function of $u_{z}$ alone. Thus, we have

$$
\begin{aligned}
S_{\mathrm{t}}(z, t)= & \rho_{\mathrm{o}}^{1 / 4} \frac{\rho_{\mathrm{e}}}{\rho_{\mathrm{o}}}\left(\frac{\partial u_{\phi}}{\partial t}\right)\left(\frac{1}{H}-\frac{\partial}{\partial z}\right) u_{z} \\
& +\rho_{\mathrm{o}}^{1 / 4} \frac{\rho_{\mathrm{e}}}{\rho_{\mathrm{o}}}\left(\frac{\partial^{2} u_{\phi}}{\partial t^{2}}\right)\left(\frac{\partial}{\partial t}\right)^{-1}\left(\frac{1}{H}-\frac{\partial}{\partial z}\right) u_{z},
\end{aligned}
$$

which is completely known when the external velocity field is specified.

The derived source function depends on both the $z$ and $\phi$ components of the turbulent velocity but does not depend explicitly on the $x$ and $y$ components of this velocity; however, the 3-D nature of turbulence is formally accounted for by using a 3-D turbulent energy spectrum to calculate the spectral tensors (see Sect. 3.7). This one-dimensional approach is consistent with the thin flux tube approximation considered in this paper. As a result of this approximation, the ratio of the tube diameter to a characteristic length scale of the turbulence is not explicitly present in the expression for the source function.

Comparison of the source function derived here to that obtained by Musielak \& Ulmschneider (2001, see their Eq. (20)) for the generation of transverse tube waves shows that gravity is not present in Eq. (23). This simply means that the generation of torsional tube waves is not effected by the presence of the fluctuating buoyancy force (Goldreich \& Kumar 1988). From a physical point of view, this is obvious as torsional oscillations of the tube are not directly effected by gravity; however, the propagation of these waves depends indirectly on gravity through stratification, which requires the tube magnetic field to diverge and, as shown in Sect. 2.3, this field divergence is responsible for the existence of the cutoff frequency. The comparison also shows that the terms depending on the second derivative of the turbulent velocity with respect to time are missing in the expression for the source function derived by Musielak \& Ulmschneider for transverse tube waves. Since these terms are of the same order of magntitude as the terms proportional to the first derivative of the turbulent velocity with respect to time, they cannot be neglected. By retaining these terms, all wave energy spectra and fluxes given by Musielak \& Ulmschneider (2001, 2002a,b) must be multiplied by a factor of 4 ; the correction is already taken into account in the transverse wave energy fluxes presented in Table 1 of this paper.

The dependence of the source function on the $\phi$-component of the turbulent velocity clearly implies that torsional tube waves are generated by turbulent motions in the $\phi$ direction. The underlying assumption here is that these motions twist the tube magnetic field at the tube surface and that once this twist occurs all remaining magnetic field lines across the tube become twisted in the same way. Here our picture is that the external turbulence occurring at all scales will not allow the tube surface to be smooth but have a rough appearance on which tangential flows will work to twist the surface field lines. This rugosity is assumed to extend into the tube such that the field lines in the tube are also twisted together with the surface fields. To simplify our analysis, we therefore assume that the turbulent motions twist the magnetic field lines at the surface without "slipping", which means that at the tube boundary $v_{\phi}=u_{\phi}$ (for 
more discussion see Sect. 2.6). The resulting torsional waves are fully described by the inhomogeneous wave Eq. (18) with the source function given by Eq. (23). By specifying the turbulent motions, we determine the form of the source function, solve the wave equation, and calculate the generation rate for torsional tube waves (see below).

\subsection{Wave equation solutions}

Since the inhomogeneous wave Eq. (18) has constant coefficients, a space and time Fourier transform can be used to obtain its solutions. By defining angular temporal, $\omega$, and spatial, $k$, frequencies, we get

$v(k, \omega)=\frac{S_{\mathrm{t}}(k, \omega)}{c_{\mathrm{A}}^{2} k^{2}-\left(\omega^{2}-\Omega_{\mathrm{T}}^{2}\right)}$,

where $k$ is the wave number in the $z$-direction, and

$S_{\mathrm{t}}(k, \omega)=\frac{1}{(2 \pi)^{2}} \iint S_{\mathrm{t}}(z, t) \mathrm{e}^{-\mathrm{i}(\omega t-k z)} \mathrm{d} z \mathrm{~d} t$

is the Fourier transform of the source function. Using Eq. (23), we may write Eq. (25) in the following form:

$$
\begin{aligned}
S_{\mathrm{t}}(k, \omega)= & \frac{\rho^{1 / 4}}{(2 \pi)^{2}} \frac{\rho_{\mathrm{e}}}{\rho_{\mathrm{o}}} \chi(k, \omega) \\
& \times \iint u_{z}(z, t) u_{\phi}(z, t) \mathrm{e}^{-\mathrm{i}(\omega t-k z)} \mathrm{d} z \mathrm{~d} t
\end{aligned}
$$

where

$\chi(k, \omega)=2 \omega\left(\frac{i}{H}+k\right)$.

Note that when $S_{\mathrm{t}}(k, \omega)=0$ the dispersion relation $\left[\left(\omega^{2}-\Omega_{\mathrm{T}}^{2}\right)-\right.$ $c_{\mathrm{A}}^{2} k^{2}=0$ ] for torsional tube waves is obtained from Eq. (24). According to this relation, the lower bound of the frequency domain for freely propagating torsional tube waves is the cutoff $\Omega_{\mathrm{T}}$; we will use this result to compute the wave energy spectra and fluxes carried by torsional tube waves.

\subsection{Wave energy spectra and fluxes}

The general expression for the MHD wave energy flux carried by Alfvén waves is $F(z, t)=-B_{\mathrm{o}} b(z, t) v(z, t) / 4 \pi$ (e.g., Anderson 1963; Musielak \& Rosner 1987). Because of the similarities between Alfvén waves and torsional tube waves (see Sect. 2.2), the wave energy flux for the latter in the $z$-direction can be written as

$F(z, t)=-\frac{B_{\mathrm{o}}}{4 \pi} b_{\phi}(z, t) v_{\phi}(z, t)$.

Substituting Eq. (10) into Eq. (28) and taking the time average yields

$<F(z, t)>_{t}=-\frac{B_{\mathrm{o}}^{2}}{4 \pi}\left\langle v_{\phi}\left(\frac{\partial}{\partial t}\right)^{-1}\left(\frac{\partial}{\partial z}\right) v_{\phi}\right\rangle_{t}$.

Using the transformation given by Eq. (17), the mean wave energy flux is

$$
\begin{aligned}
<F(z, t)>_{t}= & -\frac{B_{\mathrm{o}}^{2}}{4 \pi \rho_{\mathrm{o}}^{1 / 2}} \\
& \times\left\langle v(z, t)\left(\frac{\partial}{\partial t}\right)^{-1}\left(\frac{\partial}{\partial z}+\frac{1}{4 H}\right) v^{*}(z, t)\right\rangle_{\mathrm{t}} .
\end{aligned}
$$

The results found here are very similar in form to those obtained by Musielak \& Ulmschneider (2001) for transverse tube waves. For the benefit of readers who are unaware of the mathematical treatment previously used to derive the mean wave energy generation rate, an outline of the method is now presented. Expressing $v$ and its complex conjugate $v^{*}$ in terms of Fourier transforms and taking the time average over time scale $t_{\mathrm{o}}$ yields

$$
\begin{aligned}
<F(z, t) & >_{t}=-\frac{B_{\mathrm{o}}^{2}}{4 \pi \rho_{\mathrm{o}}^{1 / 2}} \lim _{t_{0} \rightarrow \infty} \frac{1}{t_{0}} \\
& \times \int_{-t_{0} / 2}^{+t_{0} / 2} \mathrm{~d} t \iiint \int \mathrm{d} k^{\prime} \mathrm{d} k^{\prime \prime} \mathrm{d} \omega^{\prime} \mathrm{d} \omega^{\prime \prime} v\left(k^{\prime}, \omega^{\prime}\right) \\
& \times v^{*}\left(k^{\prime \prime}, \omega^{\prime \prime}\right) \frac{-1}{\mathrm{i} \omega^{\prime \prime}}\left(\mathrm{i} k^{\prime \prime}+\frac{1}{4 H}\right) \mathrm{e}^{\mathrm{i}\left(\omega^{\prime}-\omega^{\prime \prime}\right) t-\mathrm{i}\left(k^{\prime}-k^{\prime \prime}\right) z}
\end{aligned}
$$

The time-integration is performed by using the $\delta$-function

$$
\int_{-\infty}^{+\infty} \mathrm{e}^{\mathrm{i}\left(\omega^{\prime}-\omega^{\prime \prime}\right) t} \mathrm{~d} t=2 \pi \delta\left(\omega^{\prime}-\omega^{\prime \prime}\right),
$$

and then the $\omega^{\prime \prime}$-integration is also performed analytically.

Since

$<F(z)>_{t}=\int_{-\infty}^{+\infty} \mathrm{d} \omega<F(z, \omega)>_{t}$,

the wave energy flux for a given atmospheric height $z$ and wave frequency $\omega$ is

$<F(z, \omega)>_{t_{\mathrm{o}}}=\lim _{t_{\mathrm{o}} \rightarrow \infty} \frac{B_{\mathrm{o}}^{2}}{2 t_{\mathrm{o}} \omega \rho_{\mathrm{o}}^{1 / 2} c_{\mathrm{A}}^{4}} I_{1}\left(k_{\mathrm{o}}, \omega\right) I_{2}\left(k_{\mathrm{o}}, \omega\right)$,

where

$I_{1}\left(k_{\mathrm{o}}, \omega\right)=\int_{-\infty}^{+\infty} \frac{S_{\mathrm{t}}\left(k^{\prime}, \omega\right) \mathrm{e}^{-\mathrm{i} k^{\prime} z}}{k^{\prime 2}-k_{\mathrm{o}}^{2}} \mathrm{~d} k^{\prime}$,

and

$I_{2}\left(k_{\mathrm{o}}, \omega\right)=\int_{-\infty}^{+\infty} \frac{S_{\mathrm{t}}\left(k^{\prime \prime}, \omega\right)\left(k^{\prime \prime}-i / 4 H\right) \mathrm{e}^{\mathrm{i} k^{\prime \prime} z}}{k^{\prime 2}-k_{\mathrm{o}}^{2}} \mathrm{~d} k^{\prime \prime}$,

with

$k_{\mathrm{o}}^{2}=\frac{\omega^{2}-\Omega_{\mathrm{T}}^{2}}{c_{\mathrm{A}}^{2}}$,

where for the propagating waves $k_{\mathrm{o}}^{2}$ is always positive. It is also seen that the wave energy flux must be real, therefore, the term $(-i / 4 H)$ is dropped from the calculations.

The fact that the asymptotic Fourier transforms $I_{1}$ and $I_{2}$ can be evaluated analytically for both longitudinal and transverse tube waves has been shown by Musielak et al. (1995) and Musielak \& Ulmschneider (2001), respectively. Here, we use the latter results to write

$<F(z, \omega)>_{\mathrm{t}_{\mathrm{o}}}=\lim _{t_{\mathrm{o}} \rightarrow \infty} \frac{\pi^{2}}{8 t_{\mathrm{o}}} \frac{B_{\mathrm{o}}^{2}}{\rho_{\mathrm{o}}^{1 / 2}} \frac{1}{\omega k_{\mathrm{o}} c_{\mathrm{A}}^{4}}\left|S_{\mathrm{t}}\left(k_{\mathrm{o}}, \omega\right)\right|^{2}$,

where

$\left|S_{\mathrm{t}}\left(k_{\mathrm{o}}, \omega\right)\right|^{2}=\frac{\rho_{0}^{1 / 2}}{(2 \pi)^{4}} \eta^{2}\left|\chi\left(k_{\mathrm{o}}, \omega\right)\right|^{2} \iiint \int_{\int} \mathrm{d} z \mathrm{~d} z^{\prime} \mathrm{d} t \mathrm{~d} t^{\prime}$

$\times<u_{\phi} u_{z} u_{\phi}^{\prime} u_{z}^{\prime}>\mathrm{e}^{\mathrm{i} \omega\left(t-t^{\prime}\right)-\mathrm{i} k_{0}\left(z-z^{\prime}\right)}$, 
with

$\eta=\left(\frac{\rho_{\mathrm{e}}}{\rho_{\mathrm{o}}}\right)$

and

$\left|\chi\left(k_{\mathrm{o}}, \omega\right)\right|^{2}=4 \frac{\omega^{2}}{H^{2}}\left(1+k_{\mathrm{o}}^{2} H^{2}\right)$.

Following Stein (1967), we average position and time by using

$z_{\mathrm{o}}=\frac{1}{2}\left(z+z^{\prime}\right), \quad t_{\mathrm{o}}=\frac{1}{2}\left(t+t^{\prime}\right)$,

and the relative separation and time interval quantities

$r=z-z^{\prime}, \quad \tau=t-t^{\prime}$.

Integrating over $z_{\mathrm{o}}$ and $t_{\mathrm{o}}$ yields

$$
\begin{aligned}
\left|S_{\mathrm{t}}\left(k_{\mathrm{o}}, \omega\right)\right|^{2}= & \frac{\rho_{\mathrm{o}}^{1 / 2}}{(2 \pi)^{4}} \eta^{2}\left|\chi\left(k_{\mathrm{o}}, \omega\right)\right|^{2} z_{\mathrm{o}} t_{\mathrm{o}} \\
& \times \iint \mathrm{d} r \mathrm{~d} \tau<u_{\phi} u_{z} u_{\phi}^{\prime} u_{z}^{\prime}>\mathrm{e}^{\mathrm{i}\left(\omega \tau-k_{\mathrm{o}} r\right)} .
\end{aligned}
$$

Based on the properties of isotropic and homogeneous turbulence, the fourth-order velocity correlations can be reduced to the torsional $\left(<u_{\phi} u_{\phi}^{\prime}>\right)$ and longitudinal $\left(<u_{z} u_{z}^{\prime}>\right)$ second order-correlations (see Batchelor 1960; Hinze 1975). So far, the derived wave energy flux and source function are given in the cylindrical coordinate system introduced in Sect. 2.1 to describe torsional tube waves. Now, we introduce a Cartesian coordinate system $(x, y, z)$ and use it to describe the external turbulence and calculate the second-order velocity correlations. In this new coordinate system, the turbulent velocity $u_{\phi}$ can be expressed as $u_{\phi}=-u_{x} \sin \theta+u_{y} \cos \theta$, where $\theta$ is the polar angle and the tube cross-section is assumed to be circular with radius $R_{\mathrm{t}}$ (see Fig. 1a). Because of the full symmetry between the $x$ and $y$-directions, we may assume that $\theta=90^{\circ}$ and that $u_{\phi}(z, t)=-u_{x}(z, t)$ and $u_{\phi}^{\prime}(z+r, t+\tau)=-u_{x}^{\prime}(z+r, t+\tau)$. Hence, we have $\left.<u_{\phi} u_{\phi}^{\prime}\right\rangle=<u_{x} u_{x}^{\prime}>$ and the evaluation of the secondorder velocity correlations for torsional tube waves becomes similar to that discussed by Musielak \& Ulmschneider (2001) for transverse tube waves. In Fig. 1, we show typical settings for calculating the correlation $\left\langle u_{x} u_{x}^{\prime}\right\rangle$ for the generation of both torsional and transverse tube waves.

Taking the Fourier transforms of the second-order velocity correlations defines the convolution integral

$$
\begin{aligned}
J_{\mathrm{c}}\left(k_{\mathrm{o}}, \omega\right)= & \frac{1}{(2 \pi)^{2}} \int_{-\infty}^{+\infty} \mathrm{d} r \int_{-\infty}^{+\infty} \mathrm{d} \tau R_{x x}(r, \tau) R_{z z}(r, \tau) \\
& \times \mathrm{e}^{\mathrm{i}\left(\omega \tau-k_{\mathrm{o}} r\right)},
\end{aligned}
$$

where the correlation tensors are given by

$R_{x x}(r, \tau)=<<u_{x}(z, t) u_{x}(z+r, t+\tau)>_{z}>_{t}$,

and

$R_{z z}(r, \tau)=<<u_{z}(z, t) u_{z}(z+r, t+\tau)>_{z}>_{t}$.

This allows writing the source function in the following form:

$\left|S_{\mathrm{t}}\left(k_{\mathrm{o}}, \omega\right)\right|^{2}=t_{\mathrm{o}} z_{\mathrm{o}} \frac{\rho_{\mathrm{o}}^{1 / 2}}{(2 \pi)^{2}} \eta^{2}\left|\chi\left(k_{\mathrm{o}}, \omega\right)\right|^{2} J_{\mathrm{c}}\left(k_{\mathrm{o}}, \omega\right)$
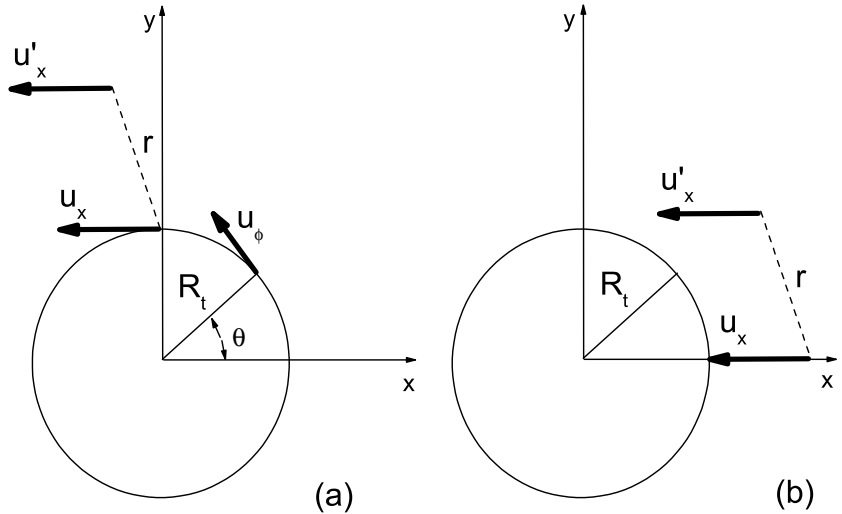

Fig. 1. Typical settings for calculating the velocity correlation $<u_{x} u_{x}^{\prime}>$ for the generation of torsional a) and transverse b) tube waves.

Since only propagating waves are considered in this paper, it is necessary to examine the source function's dependency on $k_{\mathrm{o}}$, which has been defined as the condition for freely propagating torsional tube waves (see Eq. (36)). Upon the condition $\omega \rightarrow \Omega_{\mathrm{T}}$, it is seen that $k_{\mathrm{o}} \rightarrow 0$ and the waves are evanescent. However, even for $k_{\mathrm{o}}=0$ the source function is formally not zero because $\left|\chi\left(k_{\mathrm{o}}=0, \omega\right)\right|^{2}=\omega^{2} / H^{2}$ and $J_{\mathrm{c}}\left(k_{\mathrm{o}}=0, \omega\right) \neq 0$. This implies that $\left|S_{\mathrm{t}}\left(k_{\mathrm{o}}=0, \omega\right)\right|^{2} \neq 0$ and that some wave energy is being generated at the cutoff frequency. Clearly, this energy cannot propagate to the overlying atmospheric layers and, therefore, it must be removed (see Sect. 3.2).

Finally, we combine Eqs. (37) and (47), and derive the mean wave energy generation rate [in units of $\operatorname{erg~} \mathrm{cm}^{-2} \mathrm{~s}^{-1} \mathrm{~Hz}^{-1}$ ]

$$
\begin{aligned}
<<F(\omega)>_{t_{\mathrm{o}}}>_{z_{\mathrm{o}}}= & 4 \pi \int_{\mathrm{o}}^{z_{\text {turb }}} \mathrm{d} z \frac{\rho_{\mathrm{e}}^{2}}{\rho_{\mathrm{o}}} \frac{\omega}{k_{\mathrm{o}}} \frac{\Omega_{\mathrm{T}}^{2}}{c_{\mathrm{A}}^{4}}\left(1+k_{\mathrm{o}}^{2} H^{2}\right) \\
& \times J_{\mathrm{c}}\left(k_{\mathrm{o}}, \omega\right),
\end{aligned}
$$

where $z_{\text {turb }}$ is the thickness of the turbulent region in the solar convection zone. In deriving this expression, we have taken into account the fact that only half of the total generated flux propagates upward. In Sect. 3, we use Eq. (48) to compute the wave energy spectra carried by torsional tube waves propagating upward along a given flux tube embedded in the solar atmosphere.

\subsection{Treatment of turbulence}

The correlation tensor $R_{i j}$ is defined as

$R_{i j}(\boldsymbol{r}, \tau) \equiv<<u_{i}(\boldsymbol{x}, t) u_{j}(\boldsymbol{x}+\boldsymbol{r}, t+\tau)>_{x}>_{t}$,

where the average is taken over all points $\boldsymbol{x}$ of a large volume, which can be considered infinite, and over all times $t$, which are long compared to all other time scales, and thus can also be considered infinite. A situation in which $R_{i j}$ is independent of $\boldsymbol{x}$ and $t$ is called time-independent homogeneous turbulence. Introducing the spectral tensor, $\Phi_{i j}(\boldsymbol{k}, \omega)$, and the Fourier transform $R_{i j}(\boldsymbol{r}, \tau)$, gives

$\Phi_{i j}(\boldsymbol{k}, \omega)=\frac{1}{(2 \pi)^{4}} \int \mathrm{d}^{3} r \int_{-\infty}^{+\infty} \mathrm{d} \tau R_{i j}(\boldsymbol{r}, \tau) \mathrm{e}^{\mathrm{i}(\omega \tau-\boldsymbol{k} \cdot \boldsymbol{r})}$ 
and its inverse transform

$R_{i j}(\boldsymbol{r}, \tau)=\int \mathrm{d}^{3} k \int_{-\infty}^{+\infty} \mathrm{d} \omega \Phi_{i j}(\boldsymbol{k}, \omega) \mathrm{e}^{-\mathrm{i}(\omega \tau-\boldsymbol{k} \cdot \boldsymbol{r})}$.

For homogeneous and isotropic turbulence, $R_{i j}(r, \tau)=R_{i j}(r, \tau)$ and $\Phi_{i j}(\boldsymbol{k}, \omega)=\Phi_{i j}(k, \omega)$. The correlation tensors can be evaluated when the corresponding spectral tensors are known. The spectral tensors for homogeneous and isotropic turbulence can be expressed by a 3-D turbulent energy spectrum $E(k, \omega)$, given by (Batchelor 1960)

$\Phi_{i j}(k, \omega)=\frac{E(k, \omega)}{8 \pi k^{2}}\left(\delta_{i j}-\frac{k_{i} k_{j}}{k^{2}}\right)$.

Performing the integration yields

$$
\begin{aligned}
R_{x x}(r, \tau)= & \int_{0}^{\infty} \mathrm{d} \omega \cos \omega \tau \int_{0}^{\infty} \mathrm{d} k E(k, \omega) \\
& \times\left(\frac{\sin k r}{k r}+\frac{\cos k r}{k^{2} r^{2}}-\frac{\sin k r}{k^{3} r^{3}}\right),
\end{aligned}
$$

and

$$
\begin{aligned}
R_{z z}(r, \tau)= & 2 \int_{0}^{\infty} \mathrm{d} \omega \cos \omega \tau \int_{0}^{\infty} \mathrm{d} k E(k, \omega) \\
& \times\left(\frac{\sin k r}{k^{3} r^{3}}-\frac{\cos k r}{k^{2} r^{2}}\right) .
\end{aligned}
$$

Clearly, the correlation tensors can only be calculated when the turbulent energy spectrum $E(k, \omega)$ is known. In the following, we discuss the procedure of specifying $E(k, \omega)$.

In general, there is no completely accepted theory of turbulence within the stellar convection zones. In order to describe turbulence at or below a stellar surface it is assumed that a turbulent energy spectrum, which is the distribution of energy per unit mass that is stored in eddies of different wave numbers, must be chosen (e.g., Musielak et al. 1994). The description of turbulence for our analysis is primarily phenomenological and based on two-point, two-time velocity correlation functions. Energy supplied at the largest eddy scale is dissipated by the smallest eddies thereby cascading energy through the medium size eddies. Constraining the turbulent flow to be isotropic and homogeneous, which is an idealization that may not describe correctly real flows, allow the correlation tensors (Eqs. (45) and (46)) to be computed once the form of the turbulent energy spectrum is established. For the specific purpose of this work it is a requirement to factor the turbulent energy spectrum $E(k, \omega)$ into spatial and temporal parts (Stein 1967)

$E(k, \omega)=E(k) \Delta\left(\frac{\omega}{k u_{k}}\right)$

where the average eddy velocity is given by

$u_{k}=\left[\int_{k}^{2 k} E\left(k^{\prime}\right) \mathrm{d} k^{\prime}\right]^{1 / 2}$.

The specific forms of $E(k)$ and $\Delta\left(\omega / k u_{k}\right)$ are then prescribed and used to determine the source function (Eq. (45)). Therefore, the problem of describing the external turbulent motions is reduced here to choosing the correct form of the turbulent energy spectrum.
As discussed thoroughly by Musielak et al. (1994), some modifications are needed to apply the Kolmogorov hypothesis to the turbulent motions in the solar and stellar convection zones. Defining $k_{\mathrm{t}}$ as the wave number of the energy-containing eddies and $k_{\mathrm{d}}$ as the wave number of the eddies at scales where viscous effect become important (in other words, where the turbulent cascading ends), the spatial turbulent energy spectrum $E(k)$ is given by

$E(k)= \begin{cases}0 & 0<k<0.2 k_{\mathrm{t}} \\ a \frac{u_{\mathrm{t}}^{2}}{k_{\mathrm{t}}}\left(\frac{k}{k_{\mathrm{t}}}\right) & 0.2 k_{\mathrm{t}} \leq k<k_{\mathrm{t}}, \\ a \frac{u_{\mathrm{t}}^{2}}{k_{\mathrm{t}}}\left(\frac{k}{k_{\mathrm{t}}}\right)^{-5 / 3} & k_{\mathrm{t}} \leq k \leq k_{\mathrm{d}}\end{cases}$

where the factor $a=0.758$ is determined by the normalization condition

$\int_{0}^{\infty} E(k) \mathrm{d} k=\frac{3}{2} u_{\mathrm{t}}^{2}$,

and $u_{\mathrm{t}}$ is the rms turbulent velocity defined as

$u_{\mathrm{t}}=\sqrt{\overline{u_{x}(r, t)^{2}}}=\sqrt{\overline{u_{z}(r, t)^{2}}}$.

The temporal part of the turbulent energy spectrum $\Delta\left(\omega / k u_{k}\right)$ is motivated by the Prandtl mixing length picture. This flow field is an idealization in which the turbulent eddies are in a superposition (Musielak et al. 1994). Each eddy is at a well-defined spatial scale $l$ such that the power of all eddies in that scale contribute to the power spectrum at wave number $k=2 \pi / l$. If the eddy lifetime can be approximated by a timescale such that $\tau(k) \equiv 2 \pi / k u_{k}$, where $u_{k}$ is the characteristic velocity of the eddy with wave number $k$ determined by Eq. (56), then the eddy has a unique characteristic frequency associated with it and described by $\omega(k)=2 \pi / \tau=k u_{k}$. The temporal turbulent energy spectrum would be a delta function centered at this characteristic frequency

$\Delta\left(\omega / k u_{k}\right)=\delta\left(\omega-k u_{k}\right)$.

However, it is more realistic to assume that eddies of wave number $k$ have different lifetimes. This implies that there is a distribution of eddy lifetimes. Examining the limiting behavior of Eq. (60) it is seen that the modified Gaussian turbulent frequency factor described by Musielak et al. (1994) is physically appropriate and given by

$\Delta\left(\omega / k u_{k}\right)=\frac{b}{\sqrt{\pi}\left|k u_{k}\right|} \mathrm{e}^{-\left[\left(|\omega|-\left|k u_{k}\right|\right) /\left|k u_{k}\right|\right]^{2}}$

where $b=1.78$ and is a normalization factor determined by

$\int_{0}^{\infty} \Delta\left(\omega / k u_{k}\right) \mathrm{d} \omega=1$

The results computed and presented in Sect. 3 utilize the above assumptions as well as the described spatial and temporal forms of the turbulent energy spectrum. 


\section{Results and discussion}

\subsection{Solar model}

The computer code for the solar convection zone model is a modified version of stellar envelope code used originally by Bohn (1981, 1984) and Ulmschneider et al. (1996). It is assumed that the main energy-containing eddies are comparable in size to the local pressure scale height $H$ given from a mixinglength description of convection. The mixing length parameter $\alpha=l_{\text {mix }} / H$, where $l_{\text {mix }}$ is the mixing-length, is assumed to be of order unity. The best current value for the solar convection zone presently is $\alpha=2.0$ (Trampedach et al. 1997). However, to investigate the dependence of the torsional wave generation on the mixing-length we have selected three values of the mixinglength parameter, $\alpha=1.0,1.5$ and 2.0 in our present work.

The code also requires that the surface gravity $g$ and effective temperature $T_{\text {eff }}$ are specified. In all calculations, they are taken to be $T_{\text {eff }}=5770 \mathrm{~K}$, and $g=2.736 \times 10^{4} \mathrm{~cm} \mathrm{~s}^{-2}$. Hydrogen molecule formation is included and a gray radiation transport is used. The turbulent velocity scale $u_{\mathrm{t}}$ is identified with the convective velocity of the model. Finally, it is important to note that all presented results are obtained for a single magnetic flux tube embedded in the solar convection zone. The number of magnetic flux tubes on the solar surface, or the socalled filling factor, that is, the ratio of the area covered by magnetic fields to the total surface area of the Sun, is not discussed here.

It has been shown observationally (Stenflo 1978; Solanki 1993) that at the solar surface magnetic field strengths are of the order $B_{\mathrm{o}}=1500 \mathrm{G}$. However, the equipartition magnetic field strength $B_{\text {eq }}=\sqrt{8 \pi p_{\mathrm{e}}}$, where $p_{\mathrm{e}}$ is the fluid pressure outside the tube. With $p_{\mathrm{e}}=1.17 \times 10^{5} \mathrm{dyn} \mathrm{cm}^{-2}$ (Vernazza et al. 1981) one finds the field strength $B_{\text {eq }}=1715 \mathrm{G}$. The ratio $B_{\mathrm{o}} / B_{\mathrm{eq}}=0.875$ therefore indicates a typical strength for solar magnetic flux tubes. To investigate the dependence of the wave generation rate on the magnetic field, we consider three different values of the field strength, namely, $B_{\mathrm{o}} / B_{\mathrm{eq}}=0.75,0.85$ and 0.95 .

\subsection{Propagating waves}

The cutoff frequency $\Omega_{\mathrm{T}}$ for torsional tube waves was derived for an isothermal atmosphere inside and outside the tube (see Sect. 2.1). However, models of the solar convection zone are not isothermal and the cutoff frequency does change with depth. The problem is treated here by formally dividing the tube into layers that can be considered isothermal and where $H$ and the characteristic wave velocity $c_{\mathrm{A}}$ are constant. This allows $\Omega_{\mathrm{T}}$ to be considered locally as a constant.

So far in the paper we have always assumed an exponentially spreading magnetic flux tube where one has horizontal pressure balance according to Eq. (1) where in the stable photospheric layers above the convection zone the external turbulent pressure fluctuations $\delta p_{\mathrm{t}}$ become zero. It is interesting to illustrate flux tube models which extend to the chromosphere where the horizontal pressure balance after Eq. (1) is no longer valid because neighboring flux tubes exert an

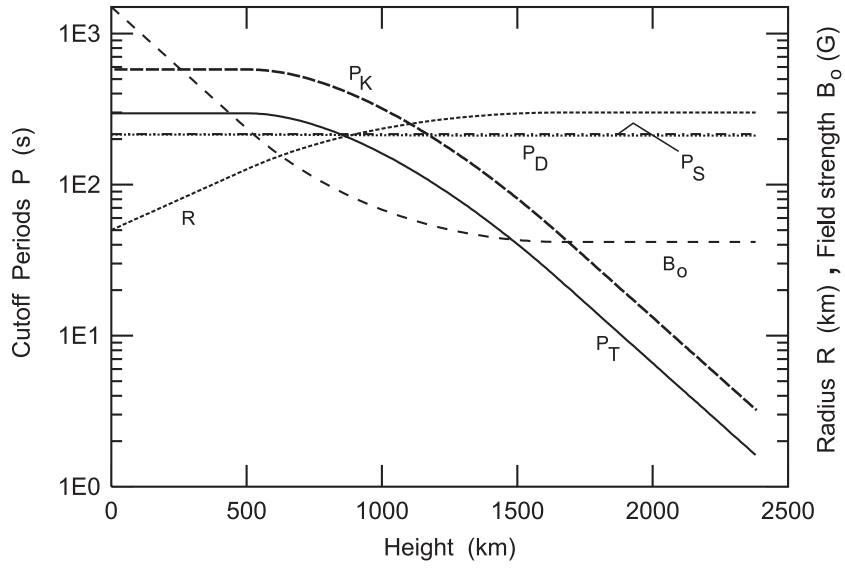

Fig. 2. Cutoff periods $P_{\mathrm{T}}$ for torsional, $P_{\mathrm{K}}$ for transverse, $P_{\mathrm{D}}$ for longitudinal and $P_{\mathrm{S}}$ for acoustic waves are plotted as a function of height in the an isothermal solar atmosphere model with $T_{\text {eff }}=5770 \mathrm{~K}$. The magnetic field strength is taken to be $B_{\mathrm{o}}=1500 \mathrm{G}$ at the bottom. The tube geometry is of a "wineglass" shape, spreading exponentially up to a height of $500 \mathrm{~km}$ and then expanding to a constant tube radius of $300 \mathrm{~km}$. The tube radius $R$ and magnetic field strength $B_{\mathrm{o}}$ are indicated.

additional external magnetic pressure to constrain the tube to a constant cross-section determined by the magnetic filling factor. Figure 2 shows such "wineglass" shaped magnetic tube model which spreads exponentially up to a height of $500 \mathrm{~km}$ and thereafter approaches a constant tube radius of $300 \mathrm{~km}$. Such tubes are thought to exist in the chromospheric network at the boundary of supergranulation cells.

We have computed the acoustic $\Omega_{\mathrm{S}}=c_{\mathrm{S}} / 2 \mathrm{H}$, longitudinal $\Omega_{\mathrm{D}}$, torsional $\Omega_{\mathrm{T}}$ and transverse $\Omega_{\mathrm{K}}=c_{\mathrm{K}} / 4 H$ cutoff frequencies by taking $B_{\mathrm{o}} / B_{\mathrm{eq}}=0.85$ and plotted in Fig. 2 the corresponding cutoff periods $P_{\mathrm{S}}=2 \pi / \Omega_{\mathrm{S}}, P_{\mathrm{D}}=2 \pi / \Omega_{\mathrm{D}}$, $P_{\mathrm{T}}=2 \pi / \Omega_{\mathrm{T}}$ and $P_{\mathrm{K}}=2 \pi / \Omega_{\mathrm{K}}$ as a function of height. It is seen that below $500 \mathrm{~km}$ height the four cutoff periods are constant because of the height-independent values of the sound speed $c_{\mathrm{S}}$ and the Alfvén speed $c_{\mathrm{A}}$ in this exponentially spreading range. Here it is seen that $P_{\mathrm{S}}$ and $P_{\mathrm{D}}$ are essentially identical because $c_{\mathrm{A}}=1.1 \times 10^{6} \mathrm{~cm} \mathrm{~s}^{-1}>c_{\mathrm{S}}=7.8 \times 10^{5} \mathrm{~cm} \mathrm{~s}^{-1}$. The kink wave cutoff period $P_{\mathrm{K}}$ is largest and the torsional wave cutoff period $P_{\mathrm{T}}$ is intermediate. This is because the height-independent kink speed $c_{\mathrm{K}}=5.9 \times 10^{5} \mathrm{~cm} \mathrm{~s}^{-1}$ is smaller than $c_{\mathrm{A}}$ which in turn is smaller that $2 c_{\mathrm{S}}$. The scale height $H=1.35 \times 10^{7} \mathrm{~cm}$ remains constant over the entire tube.

In the constant cross-section part of the tube, the magnetic field strength at $B_{\mathrm{o}}=42 \mathrm{G}$ becomes independent of height, while the density decreases rapidly with height leading to a rapidly increasing Alfvén speed and kink speed, while $c_{\mathrm{S}}$ and $c_{\mathrm{T}}$ remain constant. At $2000 \mathrm{~km}$ height one has $c_{\mathrm{A}}=5.1 \times$ $10^{7} \mathrm{~cm} \mathrm{~s}^{-1}$ and $c_{\mathrm{K}}=2.7 \times 10^{7} \mathrm{~cm} \mathrm{~s}^{-1}$. This decreases both the the torsional wave cutoff period $P_{\mathrm{T}}=8 \pi H / c_{\mathrm{A}}$ (see Eq. (19)) and the kink cutoff period $P_{\mathrm{K}}=8 \pi H / c_{\mathrm{K}}$. For some frequencies these tube waves could become trapped. Because $\Omega_{\mathrm{T}}$ is lower than $\Omega_{\mathrm{S}}$ and also lower than $\Omega_{\mathrm{D}}$, the wave energy spectra of torsional tube waves are broader than those obtained for acoustic (e.g., Ulmschneider et al. 1996) and longitudinal (Musielak et al. 2000) waves. 


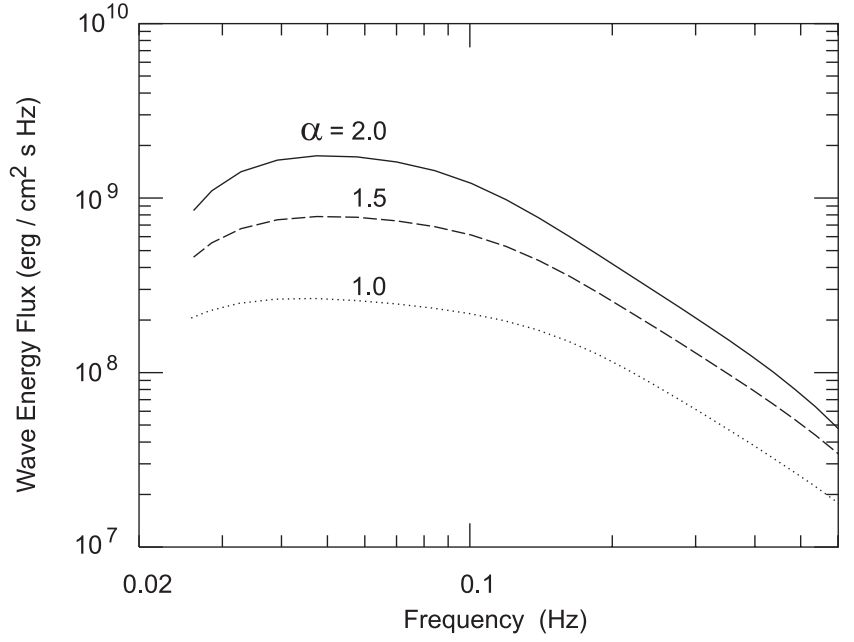

Fig. 3. Torsional tube wave energy spectra computed for different mixing-length parameters $\alpha=1.0,1.5$ and 2.0. The magnetic field strength is taken as $B_{\mathrm{o}} / B_{\mathrm{eq}}=0.85$ in all models.

Torsional tube waves are considered propagating waves if their frequencies are above the cutoff $\Omega_{\mathrm{T}}$. As already discussed in Sect. 2.6, the source function given by Eq. (47) is not equal to zero as $\omega \rightarrow \Omega_{\mathrm{T}}$. This implies that energy is being generated in the form of non-propagating (evanescent) waves. Since the non-propagating waves are not treated in this model, we eliminate them from the total energy spectrum by introducing the factor $\left(1-\Omega_{\mathrm{T}}^{2} / \omega^{2}\right)^{2}$; this factor reduces the contribution of evanescent waves to zero as $\omega \rightarrow \Omega_{\mathrm{T}}$. We utilize this factor in all computed wave energy spectra and fluxes. This guarantees that the generated torsional tube waves are always propagating and that they carry their energy away from the convection zone.

\subsection{Wave energy spectra}

The dependence of the computed wave energy spectra on the mixing-length parameter $\alpha$ is shown in Fig. 3. It is clearly seen that the torsional wave energy spectrum's overall shape is not greatly effected by the choice of this parameter, as there is no significant shift in the primary wave generation frequency domain and also the maximum remains the same.

The dependence of the wave energy spectra on the strength of the tube magnetic field is shown in Fig. 4. The presented results were obtained by taking $B_{\mathrm{o}} / B_{\mathrm{eq}}=0.75,0.85$ and 0.95 , and for a fixed value of $\alpha=2.0$. It is seen that the spectra are much broader for weak fields than for strong fields, and that the maximum shifts toward higher frequencies as the field strength $B_{0}$ increases. Both effects can be attributed to an increase in the cutoff frequency for stronger fields.

Finally, it is of some interest to compare the wave energy spectra generated by torsional tube waves to those generated by transverse and longitudinal tube waves. This comparison is shown in Fig. 5. It is seen that the rate of generation of transverse tube waves significantly exceeds that for torsional tube waves, especially, for low frequency waves. The transverse wave energy spectrum is also broader than the torsional wave and is a result of the lower cutoff frequency of the

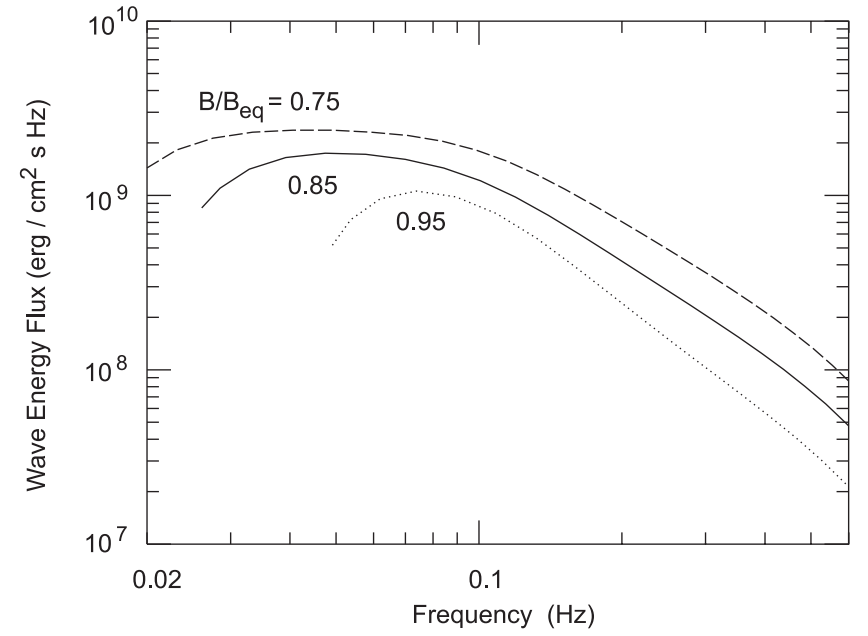

Fig. 4. Torsional tube wave energy spectra computed for three different field strengths $B_{\mathrm{o}} / B_{\text {eq }}$. The mixing-length parameter $\alpha=2$ is the same for all three cases.

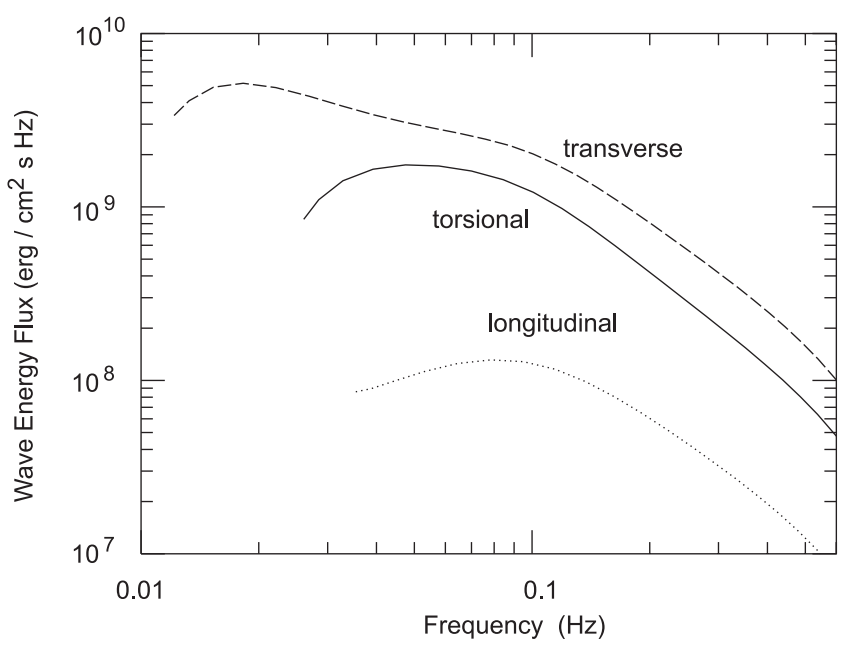

Fig. 5. Transverse, longitudinal and torsional tube wave energy spectra computed for field strengths $B_{\mathrm{o}} / B_{\mathrm{eq}}=0.85$ and the mixing-length parameter $\alpha=2$. All spectra were obtained by using analytical methods only.

transverse tube wave (see Fig. 2). The main reason for this significant difference in the efficiency of the wave excitation is the lack of contributions from the fluctuating buoyancy force to the generation of torsional tube waves. According to Musielak \& Ulmschneider (2001), this force plays the dominant role in generating transverse tube waves in the solar convection zone. It is seen that this force does not make any contribution to the excitation of torsional tube waves because these waves are not influenced (at least, in the first-order approximation) by the presence of gravity.

The results presented in Fig. 5 also show that the efficiency of generation for torsional tube waves is much higher than that for longitudinal tube waves. In addition, it is seen that the torsional wave energy spectrum is broader than the longitudinal one; again, the main difference exists for low frequency waves because the cutoff period for torsional tube waves is lower than the corresponding one for longitudinal tube waves 
Table 1. Total wave energy fluxes $F_{\text {tors }}\left(\mathrm{erg} / \mathrm{cm}^{2} \mathrm{~s}\right)$ generated as torsional waves in a single magnetic flux tube embedded in the solar convection zone are compared to the fluxes for transverse tube waves, $F_{\text {trans }}$ and longitudinal tube waves, $F_{\text {long }}$; note that the factor 4 (see discussion below Eq. (23)) was included in the computation of the wave energy fluxes for transverse tube waves. All presented fluxes were computed by using analytical methods only.

\begin{tabular}{ccccc}
\hline \hline$\alpha$ & $B_{\mathrm{o}} / B_{\mathrm{eq}}$ & $F_{\text {tors }}$ & $F_{\text {trans }}$ & $F_{\text {long }}$ \\
\hline 1.0 & 0.85 & $6.0 \times 10^{7}$ & $1.2 \times 10^{8}$ & $6.3 \times 10^{6}$ \\
1.5 & 0.85 & $1.4 \times 10^{8}$ & $2.9 \times 10^{8}$ & $1.4 \times 10^{7}$ \\
2.0 & 0.85 & $2.6 \times 10^{8}$ & $5.6 \times 10^{8}$ & $2.4 \times 10^{7}$ \\
2.0 & 0.75 & $4.4 \times 10^{8}$ & $8.8 \times 10^{8}$ & $5.5 \times 10^{7}$ \\
2.0 & 0.95 & $1.3 \times 10^{8}$ & $3.6 \times 10^{8}$ & $5.9 \times 10^{6}$ \\
\hline
\end{tabular}

(see Fig. 2). The differences become important when the role played by each one of these waves in the atmospheric heating is investigated; we briefly discuss this problem in the following subsection.

\subsection{Energy fluxes}

Considering a single magnetic flux tube, the total torsional wave energy flux is computed by integrating the wave energy spectra over the range $\Omega_{\mathrm{T}} \leq \omega \leq 25 \Omega_{\mathrm{T}}$. The results are presented in Table 1, which shows that for the same value of $\alpha$ and $B_{\mathrm{o}} / B_{\text {eq }}$ transverse tube waves are two times more efficiently generated than torsional tube waves; however, the efficiency of the excitation of the latter is one order of magnitude higher than longitudinal tube waves. These are expected results as it is much easier for the external turbulent motions to shake and twist the tube than to twist it (e.g., Spruit \& Roberts 1983; Musielak \& Ulmschneider 2001).

From the results presented in Table 1, one also finds that the $\alpha$-dependence scales with the efficiency of the wave generation process: the higher the efficiency the stronger is the $\alpha$ dependence. Since higher values of $\alpha$ give larger convective velocities, the effect is stronger for the waves that are easier to generate. It is seen that the wave energy flux for a mixinglength parameter, $\alpha=2.0$ is 4.4 times larger than for $\alpha=1.0$; this increase is caused by much higher convective velocities in the former case than in the latter. The approximate dependence on the parameter $\alpha$ is found to be

$$
F_{\text {tors }} \approx 6.0 \times 10^{7} \alpha^{2.1} \mathrm{erg} \mathrm{cm}^{-2} \mathrm{~s}^{-1} \text {. }
$$

This $\alpha$-dependence can be compared to that obtained for transverse $\left(\sim \alpha^{2.4}\right)$ and longitudinal $\left(\sim \alpha^{1.8}\right)$ tube waves by Musielak et al. (2001) and Musielak et al. (2000), respectively.

According to the results given in Table 1, one sees that the torsional wave energy flux decreases with increasing magnetic field strength $B_{0}$. An approximate fitting to these data yields the following $B_{0}$-dependence

$$
F_{\text {tors }} \approx 1.0 \times 10^{8}\left(B_{\mathrm{o}} / B_{\mathrm{eq}}\right)^{-4.9} \mathrm{erg} \mathrm{cm}^{-2} \mathrm{~s}^{-1} \text {. }
$$

By comparing this dependence to that previously derived for transverse $\left(\sim B_{\mathrm{o}}^{-3.6}\right)$ and longitudinal waves $\left(\sim B_{\mathrm{o}}^{-9.4}\right)$, one clearly sees that the efficiency of the generation of longitudinal tube waves depends very strongly on the strength of the tube magnetic field. The affect is also important for the generation of torsional tube waves and transverse tube waves, however transverse waves are the least effected.

The fact that the efficiency of generation of different tube waves depends so differently on the strength of the tube magnetic field can easily be explained by the role played by "stiffness" of magnetic flux tubes in the wave generation. This "stiffness" is obviously greater for stronger magnetic field strength (for example, it is much greater for $B_{\mathrm{o}} / B_{\mathrm{eq}}=0.95$ than for $\left.B_{\mathrm{o}} / B_{\text {eq }}=0.75\right)$. The "stiffer", the more resistant the tube becomes to external motions. The fact that the longitudinal wave generation is more affected than the other processes is directly related to the amount of gas inside the tube: the stronger the field, the less gas one has inside and the more difficult it is to excite the wave. In the case of torsional waves, the strength of the field determines how difficult it is for the external motions to twist the tube. This is similar in the generation of transverse tube waves where stiffer fields inhibit bending by the same weak external turbulent flows. However, because of their high efficiency of generation they are less effected. In summary, it is harder to twist the magnetic flux tubes than shake them but it is easier to twist the tube than to squeeze it. In other words, one finds that the lower the efficiency of the wave generation the stronger the dependence on the tube magnetic field.

\subsection{The role of torsional tube waves in atmospheric heating}

We have computed the wave energy spectra and fluxes carried by torsional tube waves in the solar atmosphere, and compared them to those previously obtained for longitudinal and transverse tube waves. Our approach is analytical, which means that the calculated fluxes represent only lower bounds for the realistic fluxes. The obtained results show that torsional tube waves propagating in the solar atmosphere carry less energy than transverse tube waves but more energy than longitudinal tube waves. This implies that torsional tube waves could significantly contribute to the atmospheric heating and that the energy fluxes carried by these waves must be included into theoretical models of stellar chromospheres and coronae.

The problem of heating stellar chromospheres and coronae is not yet fully understood despite significant observational and theoretical progress (e.g., Narain \& Ulmschneider 1996, and references therein). As already mentioned in Sect. 1, recent results obtained by Ulmschneider et al. (2001) and Fawzy et al. (2002a,b) clearly show that the wave heating mechanism based on longitudinal and transverse tube waves can explain the observed level of stellar activity in late-type stars only up to the level of the Ca II $H$ and $K$ line formation, while to explain the emission of the higher chromospheric layers, where the $\mathrm{Mg} \mathrm{II} \mathrm{h}$ and $\mathrm{k}$ lines originate, another non-wave magnetic (e.g., reconnective) heating mechanism seems to be required.

The results presented in this paper demonstrate that the contribution of torsional tube waves to solar and stellar atmospheric heating can be important because the amount of energy carried by these waves significantly exceeds the amount 
of energy dissipated by longitudinal tube waves. Even though, torsional waves, carrying more energy than longitudinal waves but appreciably less than transverse waves, are found to be difficult to damp similarly as the transverse waves. However, for both wave modes energy dissipation can occur via nonlinear mode-coupling to longitudinal tube waves (for transverse waves, see Zhugzhda et al. 1995, and for torsional waves, see Hollweg et al. 1982). These mode-couplings are particularly efficient when in the transverse wave case kink shocks and in the torsional wave case switch-on shocks occur. Therefore, the amount of energy carried by torsional and transverse tube waves should be included into the theoretical models of stellar chromospheres, and the efficiency of mode-coupling studied.

\section{Conclusions}

The following conclusions can be drawn from the analytic study of linear torsional wave generation in magnetic flux tubes embedded in the solar convection zone.

1. Torsional magnetic tube waves have a cutoff frequency $\Omega_{\mathrm{T}}=c_{\mathrm{A}} / 4 H$ where $c_{\mathrm{A}}$ the Alfvén velocity and $H$ the scale height. Typical values of the cutoff periods $P_{\mathrm{T}}=2 \pi / \Omega_{\mathrm{T}}$ are 100 to $300 \mathrm{~s}$. Long period torsional waves could show trapping due to this cutoff frequency.

2. The typical wave energy flux carried by torsional tube waves in the solar atmosphere is approximately $2 \times$ $10^{8} \mathrm{erg} / \mathrm{cm}^{2} \mathrm{~s}$. This is one order of magnitude more than the longitudinal wave energy flux and 2 times less than the transverse wave energy flux. This comparison is based on the wave energy fluxes obtained by using analytical methods only.

3. For different values of the mixing length parameter $\alpha$, the shapes of the computed wave energy spectra remain practically the same. However, the amount of generated wave energy strongly increases with increasing $\alpha$.

4. The computed wave energy spectra and fluxes are sensitive to the strength of the tube magnetic field. For weak fields, the spectra are much broader than for strong fields - this is caused by the cutoff frequency dependence of the magnetic field. The total amount of energy carried by torsional tube waves decreases when the strength of the tube magnetic field is increased.

5. The total amount of energy carried by torsional tube waves does not depend as strongly on the strength of the tube magnetic field as the longitudinal wave energy fluxes. The difference can be easily explained due to the role played by the "stiffness" of flux tubes in the process of wave excitation.

6. Our results are only valid for linear generation of torsional tube waves. Therefore, the presented wave energy fluxes must be regarded as lower bounds for realistic energies carried by these waves. Despite this, the fluxes carried by torsional tube waves are likely to play important role in solar and stellar atmospheric heating.

Acknowledgements. This work was supported by NSF under grant ATM-0087184 (Z.E.M., M.N. and P.U.), by the DFG grant U157/253 , and by NATO under grant CRG-910058 (P.U. and Z.E.M.).
Z.E.M. also acknowledges the support of this work by the Alexander von Humboldt Foundation.

\section{References}

Anderson, J. E. 1963, Magnetohydrodynamic Shock Waves (Cambridge: MIT Press), 50

Batchelor, G. K. 1960, Theory of Homogeneous Turbulence (Cambridge: Cambridge University Press), 49

Buchholz, B., Ulmschneider, P., \& Cuntz, M. 1998, ApJ, 494, 700

Bohn, H. U. 1981, Ph.D. Thesis, Univ. Würzburg, Germany

Choudhuri, A. R., Auffret, H., \& Priest, E. R. 1993a, Sol. Phys., 143, 49

Choudhuri, A. R., Dikpati, M., \& Banerjee, D. 1993b, ApJ, 413, 811

Cuntz M., Rammacher W., Ulmschneider P., Musielak Z. E., \& Saar S. H. 1999, ApJ, 522, 1053

Cuntz M., Ulmschneider P., \& Musielak Z. E. 1998, ApJ, 493, L117

Defouw, R. J. 1976, ApJ, 209, 266

Fawzy, D. E., Rammacher, W., Ulmschneider, P., Musielak, Z. E., \& Stępień, K. 2002a, A\&A, 386, 971

Fawzy, D.E., Ulmschneider, P., Stępień, K., Musielak, Z.E., \& Rammacher, W. 2002b, A\&A, 386, 983

Goldreich, P., \& Kumar, P. 1988, ApJ, 326, 462

Hinze, J. O. 1975, Turbulence (New York: McGraw Hill), 61, 202

Hollweg, J. V., Jackson, S., \& Galloway, D. 1982, Sol. Phys., 75, 35

Huang P., Musielak, Z. E., \& Ulmschneider, P. 1995, A\&A, 279, 579

Lighthill, M. J. 1952, Proc. Roy. Soc. London A, 211, 564

Muller, R. 1989, in Solar and Stellar Granulation, ed. R. J. Rutten \& G. Severino (Dordrecht: Kluwer Academic Publ.), 101

Muller, R., Roudier, Th., Vigneau, J., \& Auffret, H. 1994, A\&A, 283, 232

Musielak, Z. E., \& Rosner, R. 1987, ApJ, 315, 371

Musielak, Z. E., Rosner, R., Gail, H. P., \& Ulmschneider, P. 1995, ApJ, 448,865

Musielak, Z. E., Rosner, R., Stein, R. F., \& Ulmschneider, P. 1994, ApJ, 423, 474

Musielak, Z. E., Rosner, R., \& Ulmschneider, P. 1989, ApJ, 337, 470

Musielak, Z. E., Rosner, R., \& Ulmschneider, P. 2000, ApJ, 541, 410

Musielak, Z. E., Rosner, R., \& Ulmschneider, P. 2002, ApJ, 573, 418

Musielak, Z. E., \& Ulmschneider, P. 2001, A\&A, 370, 541

Musielak, Z. E., \& Ulmschneider, P. 2002a, A\&A, 386, 606

Musielak, Z. E., \& Ulmschneider, P. 2002b, A\&A, 386, 615

Narain U., \& Ulmschneider, P. 1996, Space Sci. Rev., 75, 453

Roberts, B., \& Ulmschneider, P. 1997, in Solar and Heliospheric Plasma Physics, ed. G. M. Simett, C. E. Alissandrakis, \& L. Vlahos (Berlin: Springer Verlag), 75

Saar, S. H. 1996, in Stellar Surface Structure, ed. K. Strassmeier, \& J. L. Linsky (Kluwer), IAU Symp., 176, 237

Solanki, S. K. 1993, Space Sci. Rev., 63, 1

Spruit, H. C. 1981, A\&A, 98, 155

Spruit, H. C. 1982, Sol. Phys., 75, 3

Spruit, H. C., \& Roberts, B. 1983, Nature, 304, 401

Stein, R. F. 1967, Sol. Phys., 2, 385

Trampedach, R., Christensen-Dalsgaard, J., Nordlund, A., \& Stein, R. 1997, 73

Ulmschneider P., Zähringer K., \& Musielak, Z. E. 1991, A\&A, 241, 625

Ulmschneider, P., Theurer, J., \& Musielak, Z. E. 1996, A\&A, 315, 212

Ulmschneider, P., \& Musielak, Z. E. 1998, A\&A, 338, 311

Ulmschneider, P., Musielak, Z. E., \& Fawzy, D. E. 2001, A\&A, 374, 662

Ulmschneider, P., Fawzy, D. E., Musielak, Z. E., \& Stępień, K. 2001, ApJ, 559, L167

Vernazza, J. E., Avrett, E. H., \& Loeser, R. 1981, ApJS, 45, 635

Zhugzhda, Y. D., Bromm, V., \& Ulmschneider, P. 1995, A\&A, 300, 302 